\title{
Manejo laparoscópico de un teratoma quístico maduro bilateral Reporte de un caso
}

\author{
Juan Diego Villegas, MD*; Felipe Arbeláez, MD*; Angela María Ruiz, MD ${ }^{\circ}$
}

RESUMEN: El manejo laparoscópico de las masas anexiales benignas se ha difundido recientemente. La mayor experiencia de los cirujanos y el diagnóstico imagenológico certero, han hecho que cada vez más las pacientes puedan beneficiarse del manejo laparoseópico, con las conocidas ventajas de costo, tiempo de hospitalización, tipo de abordaje y tiempo de incapacidad.

Se presenta aquí el resumen de una paciente con teratomas quísticos maduros bilaterales manejada por vía laparoscópica en la Unidad de Endoscopia Gineco-Obstétrica (UEGO) del Hospital Infantil Universitario Lorencita Villegas de Santos de Santafé de Bogotá.

PALABRAS CLAVES: Teratoma quístico benigno, laparoscopia.

SUMMARY: The laparoscopic management of the benign adnexal masses is progressively more frequent. Maybe the more surgeon's experience and the images technique accuracy have let the patients to profit of the laparoscopic management, taking advantage of all the laparoscopic surgery benefits.

Here is a case report of a patient with bilateral benign cystic teratoma who went to laparoscopic management at the Hospital Infantil Universitario Lorencita Villegas de Santos in Santafé de Bogotá.

KEY WORDS: Benign cystic teratoma, laparoscopy.

\section{Introducción}

Los teratomas quísticos maduros son las neoplasias ováricas más frecuentes en mujeres en la segunda y tercera década de la vida.

Su presentación clínica va desde las pacientes asintomáticas hasta las que consultan por un abdomen agudo debido a la torsión del tumor, fenómeno que se presenta con mayor frecuencia durante el embarazo.

Su tratamiento depende de múltiples factores, principalmente las características de la masa y el deseo de paridad de la paciente.

A continuación presentamos el resumen de historia clínica de una paciente con teratomas quísticos maduros bilaterales que fue manejada por la Unidad de Endoscopia Ginecológica de nuestro hospital.

\section{Resumen de historia clínica}

Se trata de una paciente de 34 años, natural y procedente de Bogotá. Soltera. Consultó por cuadro de dolor pélvico crónico de seis meses de evolución, sin sintomatología asociada, eventos previos o tratamiento.

La paciente niega antecedentes familiares. Los antecedentes gineco-obstétricos muestran ciclos regulares.

Residente III año HILVdeS.

Jefe de la Unidad de Endoscopia GinecoObstétrica HILVdeS.

Instructora Unidad de Endoscopia GinecoObstétrica HILVdeS.
G4P3A1V3. Paridad satisfecha. El resto de antecedentes personales son negativos.

Al examen físico el único hallazgo positivo se encuentra al tacto vaginal en donde a nivel del anexo derecho se palpa masa móvil, no adherida, no dolorosa, renitente, de $5 \times 5 \mathrm{~cm}$. Anexo izquierdo normal.

Dentro del estudio prequirúrgico la paciente tiene marcadores tumorales normales (AFP 2.0 y BHCG 2.6) y eco transabdominal-transvaginal que muestra en el anexo derecho una masa quística de $6.4 \times 5.7 \times 5.1 \mathrm{~cm}$ (volumen $97 \mathrm{cc}$ ) con material heterogéneo más espeso en el contorno superior, sin vegetaciones ni tabiques. Fondos de saco libres (Figura 1).

Se programa para laparoscopia operatoria y biopsia por congelación.

En el procedimiento no se encuentra ascitis. Superficies peritoneales, apéndice, hígado, vesícula y estómago de características normales. Útero de características normales. Ovario derecho: Masa $7 \mathrm{~cm}$ diámetro móvil y libre (\#1). Cápsula lisa, nacarada, sin papilas externas. Líquido del quiste hemorrágico. Cistoscopia sin papilas. En el borde inferior del ovario derecho se encuentra otra masa de predominio sólido de $4 \mathrm{~cm}$ diámetro, irregular, con líquido viscoso y pelos en su interior (\#2). Anexo izquierdo involucrado en proceso adherencial severo, en donde el ovario tenía lesión quística de $3.5 \mathrm{~cm}$ de diámetro, lisa, sin papilas, con pelos y contenido sebáceo en su interior (\#3). 
Figura 1

QUISTE DEL CUERPO LUTEO

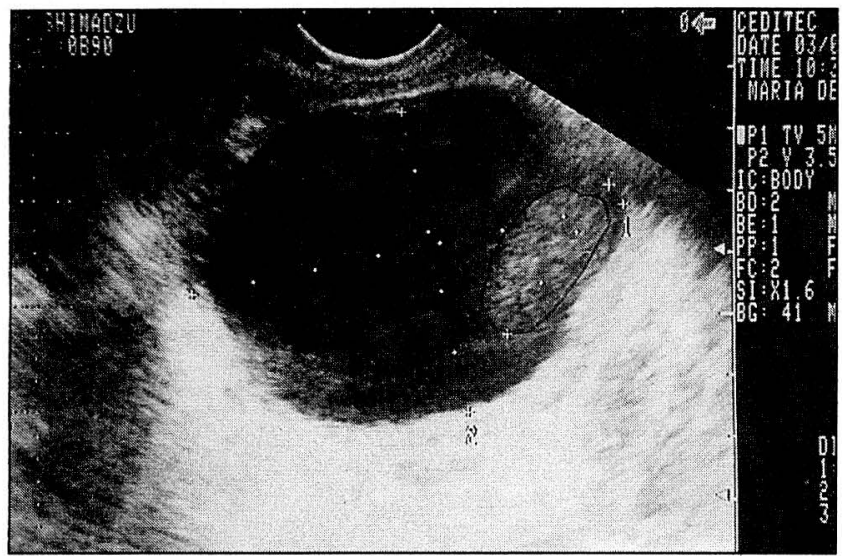

Figura 2

TERATOMA QUISTICO MADURO OVARIO DERECHO

IMAGEN LAPAROSCOPICA

Figura 3

TERATOMA BENIGNO OVARIO IZQUIERDO CON TEJIDO OSEO Y PELOS

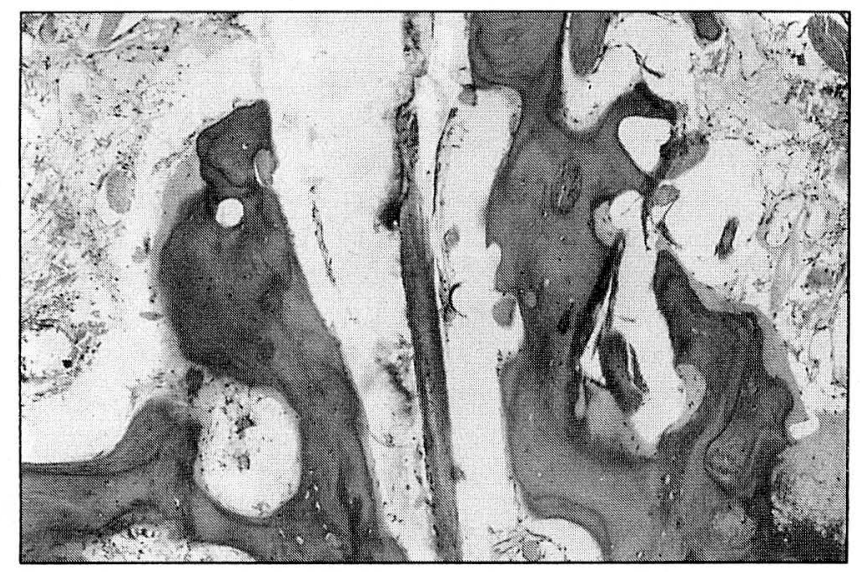

Se practicó lavado y citología peritoneal. Punción, citología, fenestración y cistoscopia de las masas descritas. Se envió muestra del tejido de las tres masas a biopsia por congelación, que mostró:

1 Quiste del cuerpo lúteo

2 Teratoma quístico maduro ovario derecho

3 Teratoma benigno ovario izquierdo con Tejido óseo y pelos.

Se practicó anexectomía bilateral con energía bipolar y corte. Se utilizaron endobolsas logrando extraer la piezas quirúrgicas sin derramar el contenido de los teratomas en la cavidad peritoneal (Figura 2)

Las citologías peritoneal y de los líquidos de las lesiones quísticas fueron todas negativas. El resultado definitivo de anatomía patológica confirmó el de la biopsia por congelación (Figura 3).

La paciente fue dada de alta 12 horas después del procedimiento.

La evolución postoperatoria inmediata fue adecuada y $\sin$ complicaciones.

Asistió a control postoperatorio refiriendo sintomatología hipoestrogénica.

Se solicitó estudio paraclínico previo a inicio de terapia de suplencia hormonal, la que viene recibiendo desde hace 2 meses con adecuada tolerancia.

\section{Discusión}

Los teratomas ováricos son neoplasias de las células germinales. Pueden ser de tres tipos:

1. Inmaduro Sólido

Quístico

Mixto

2. Maduro Sólido

Quístico

a. Teratoma quístico maduro (quiste dermoide)

b. Quiste dermoide con transformación maligna

3. Altamente especializado (monodérmico)

Struma Ovarii

Carcinoide

Struma ovarii y carcinoide

Otros

Son responsables del $15 \%$ de todas las neoplasias ováricas y el $95 \%$ de estos corresponden a teratomas quísticos maduros.

Los teratomas quísticos maduros son los tumores ováricos mas frecuentes en mujeres entre la segunda y tercera década de la vida. El contenido mas frecuente de estas neoplasias es de origen ectodérmico (quistes dermoides verdaderos) -piel, folículos pilosos, glándulas sebáceas y sudoríparas, etc.-, pero pueden contener estructuras derivadas del mesodermo y endodermo.

La degeneración maligna de estos tumores ocurre en menos del 3\%, generalmente en pacientes postmenopausicas, y usualmente lo hacen hacia un tipo histológico escamoso (1).

Pueden afectar a pacientes embarazadas hasta en el $12 \%$ de los casos, en los cuales la ruptura es mas frecuente $(2-4)$. 
Clínicamente se caracterizan por ser lesiones de crecimiento lento que se presentan por los síntomas relacionados con el tamaño de la masa, síntomas compresivos, torsión y, ocasionalmente, por la peritonitis química producto de la ruptura de los quistes y el vaciamiento de su contenido a la cavidad peritoneal.

La bilateralidad en este tipo de neoplasias alcanza el 10 a $25 \%$ de los casos (1).

Hace unos años, la cuña ovárica contralateral se recomendó en todas las pacientes, pero la experiencia actual muestra que no es necesaria cuando el ovario del lado contrario es macroscópicamente sano.

El estudio prequirúrgico debe ser cuidadoso y esquemático, para que, al momento del abordaje, el diagnóstico sea lo mas cercano posible y las posibilidades de malignidad sean reducidas al máximo (5).

Los hallazgos ecográficos son muy significativos y pueden sugerir el diagnóstico en un porcentaje alto. Algunos autores informan una especificidad del $99 \%$ de la ecografía transvaginal en el diagnóstico de teratomas quísticos maduros (6-8).

El tratamiento de este tipo de neoplasias depende de las características del tumor y los deseos de paridad de la paciente.

Siempre que se haya descartado malignidad y se haya inspeccionado la cavidad pélvica y abdominal de forma cuidadosa, la decisión del tratamiento quirúrgico depende de las características de la masa y la historia clínica de la paciente. La cistectomía puede intentarse sobre todo en las lesiones bilaterales, de menos de $5 \mathrm{~cm}$ y en pacientes que no han satisfecho su paridad. En las lesio- nes que no cumplan estas características, la ooforectomía es el tratamiento de elección $(2,5,9-11)$.

El acercamiento terapéutico de este tipo de tumores por vía laparoscópica es cada día más frecuente. La única consideración que estadísticamente influye en el manejo por laparotomía es el tamaño de la masa. La edad de la paciente, antecedentes quirúrgicos o paridad no son variables significativas en la toma de decisión de la vía de abordaje (10).

La gran mayoría de las series que reportan manejo laparoscópico de los teratomas quísticos benignos y las que comparan el manejo por laparoscopia con la laparotomía concluyen que la vía endoscópica es útil, segura y eficaz en el tratamiento de estas lesiones. En la tabla 1 se muestran los principales trabajos de los grupos que mas experiencias tienen en el manejo de los teratomas quísticos benignos por vía laparoscópica.

Se han descrito complicaciones tempranas y tardías del manejo quirúrgico laparoscópico de los quistes dermoides (12-13). La ruptura intraoperatoria del quiste puede producir peritonitis química granulomatosa por el esparcimiento del contenido sebáceo en la cavidad peritoneal. La aparición de sintomatología suele ser aguda, pero puede ser tan tardía como 18 meses postoperatoria, en la que el dolor y las adherencias son el hallazgos cardinal. La irrigación exhaustiva de la cavidad abdominal y el uso de endobolsas evita, según las series de la literatura, ésta complicación (12-13).

Ulrich y Keckstein publicaron en 1996 una revisión de 70 teratomas quístico maduros manejados por laparoscopia. En 8 de los casos de manejo conservador,

Tabla 1

MANEJO LAPAROSCOPICO DE TERATOMAS QUISTICOS BENIGNOS DE OVARIO

\begin{tabular}{|lcll|}
\hline Autor y año & No. casos & Características especiales & Complicaciones o comentarios \\
\hline Reich\&McGlynn 1992(13) & 27 & Uso de endobag & $\begin{array}{l}\text { Laceración V. } \\
\text { Cava con trocar }\end{array}$ \\
Nezhat\&Winer 1989(12) & 9 & Uso de lavado masivo de cavidad & Ninguna \\
Kutteh\&Albert 1991 (14) & 1 & $\begin{array}{l}\text { Reporte de un caso } \\
\text { asociado a E. Ectópico }\end{array}$ & Ninguna \\
Parker 1996(3) & 12 & $\begin{array}{l}\text { Todos los procedimientos } \\
\text { en embarazadas }\end{array}$ & Ninguna \\
Albini\&Benadiva 1993(9) & 38 & $\begin{array}{l}\text { Comparación laparotomía } \\
\text { Vs. Laparoscopia }\end{array}$ & $\begin{array}{l}\text { Ninguna. No } \\
\text { diferencias entre grupos }\end{array}$ \\
Howard 1995(10) & 20 & $\begin{array}{l}\text { Comparación laparotomía } \\
\text { Vs Laparoscopia }\end{array}$ & $\begin{array}{l}\text { Ninguna. No } \\
\text { diferencia entre grupos }\end{array}$ \\
Nitke\&Goldman 1996(15) & 38 & $\begin{array}{l}\text { Comparación laparotomía } \\
\text { Vs Laparoscopia }\end{array}$ & $\begin{array}{l}\text { Ninguna. No } \\
\text { diferencia entre grupos }\end{array}$ \\
Canis\&Mage 1994(11) & 141 & Experiencia 12 años & Informe de 819 masas \\
Ulrich\&Keckstein 1996(16) & 70 & $\begin{array}{l}\text { 60 cistectomías } \\
\text { 10 anexectomías 8 second look }\end{array}$ & Ninguna \\
\hline
\end{tabular}


las pacientes fueron llevadas a segunda mirada laparoscópica. El estudio concluyó que el uso de endobolsas y el lavado exhaustivo de la cavidad abdominal, en los casos en los que se derramó el contenido del quiste en la cavidad peritoneal, evita las adherencias y la posibilidad de peritonitis química granulomatosa (16).

En conclusión, el manejo de las masas ováricas benignas y en especial de los teratomas quísticos maduros tiende a variar con el paso del tiempo. La laparoscopia se ha ido convirtiendo en una técnica, eficaz, confiable, segura y útil para el manejo de este tipo de patologías, añadiéndole al tratamiento adecuado, las ventajas del menor costo y tiempo de hospitalización, mejor resultado estético, buena tolerancia del procedimiento y menor tiempo de incapacidad laboral.

\section{BIBLIOGRAFIA}

1. DiSaia PJ., Creasman WT. Clinical Gynecologic Oncology. 4th. Ed. Mosby-Year book inc. 1993; 426-466.

2. Kadar N. Atlas of Laparoscopic Pelvic Surgery. 1st Ed. Blackwell science; 1995 ; 137-146.

3. Parker WH., Childers JM., Canis M et al. Laparoscopic Management of benign cystic teratomas during pregnancy. Am J Obstet Gynecol 1996; 174: 1499

4. Howard MF., Vill M. Laparoscopic adnexal surgery during pregnancy. J Am Assoc Gynecol Laparose 1994; 2: 91.

5. Curtin JP. Management of the adnexal mass. Gynecol Oncol 1994; 55: $\mathrm{S} 42$.

6. Mais V., Guerriero S., Ajossa S et al. Transvaginal ultrasonography in the diagnosis of cystic teratoma. Obstet Gynecol 1995; 85: 48.

7. Caspi B., Elchalal U et al. Preoperative sonography in detecting small benign cystic teratomas. Int J Gynaecol Obstet 1995; 48: 75.

8. Sassone AM., Timor-tritsh IE et al. Transvaginal sonographic caracterization of ovarian disease: Evaluation of a new scoring system to predict ovarian malignancy. Obstet Gynecol 1991; 78: 70.
9. Albini SM., Benadiva CA., Haven K et al. Management of benign ovarian cystic teratomas: Laparoscopy compared with laparotomy. Am J Obstet Gynecol 1994; 24: 221.

10. Howard FM. Surgical Management of benign cystic Teratoma: Laparoscopy versus laparotomy. J Reprod Med 1995; 40: 495.

11. Canis M., Mage G., Pouly JL et al. Laparoscopic diagnosis of adnexal masses: A 12-year experience with long-term follow-up. Obstet Gynecol 1994; 83: 707.

12. Nezhat C., Winer WK., Nezhat F. Laparoscopic removal of dermoid cysts. Obstet Gynecol 1989; 73: 278.

13. Reich H., McGlyn F., Taylor P. Laparoscopic management of ovarian dermoid cysts. J Reprod Med 1992; 37: 640.

14. Kutteh WH., Albert T. Mature cystic teratoma of the fallopian tube associated with an ectopic pregnancy. Obstet Gynecol 1991; 78: 984.

15. Nitke S., Goldman GS., Fish B et al. The management of dermoid cysts: A comparative study of laparoscopy and laparotomy. Isr J Med Sci 1996; 32: 1177.

16. Ulrich U., Keckstein J et al. Endoscopic surgery for mature teratoma of the ovary. Surg Endosc 1996;10: 900.

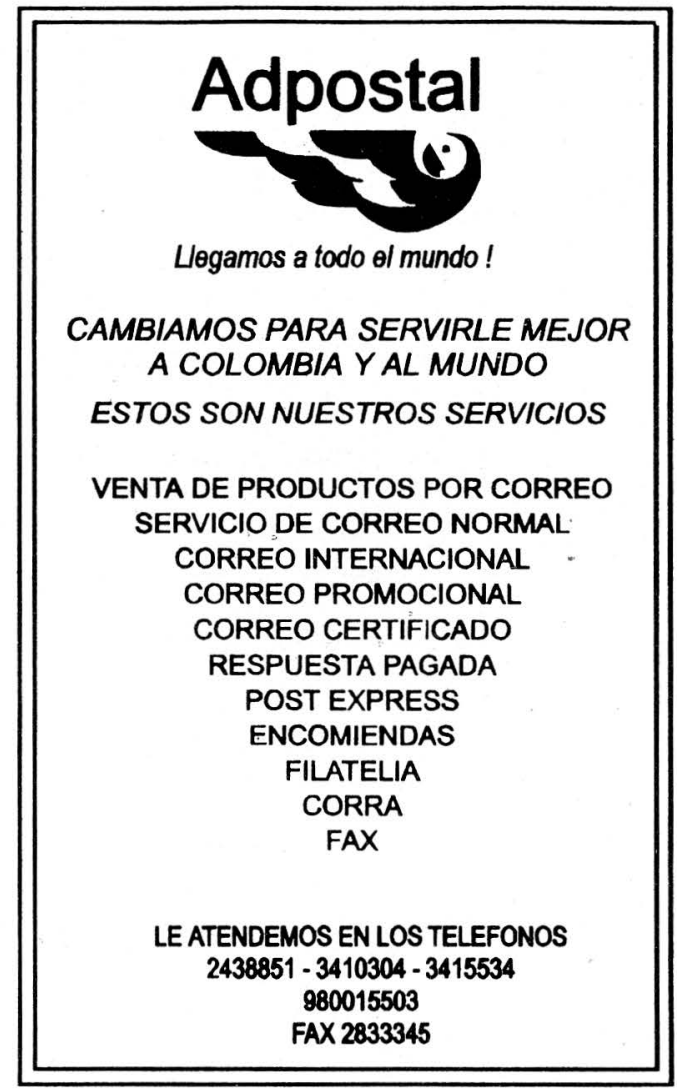

